\title{
Towards a Social Justice Framework of Mental Health Recovery
}

\author{
MARINA MORROW \\ Faculty of Health Sciences, Centre for the Study of Gender, Social Inequities and Mental \\ Health, Simon Fraser University
}

\author{
JULIA WEISSER ${ }^{1}$ \\ Centre for the Study of Gender, Social Inequities and Mental Health \\ Simon Fraser University
}

\begin{abstract}
In this paper we set out the context in which experiences of mental distress occur with an emphasis on the contributions of social and structural factors and then make a case for the use of intersectionality as an analytic and methodological framework for understanding these factors.

We then turn to the political urgency for taking up the concept of recovery and argue for the importance of research and practice that addresses professional domination of the field, and that promotes ongoing engagement and dialogue about recovery as both a personal and social experience. To this end, we describe a unique project that sought to deepen our understanding of how recovery is being thought about and applied in the current context of mental health care in Vancouver, BC, with a specific focus on how, and whether, people are taking up and addressing dimensions of power that we see as critical to the operationalization of recovery within a social justice framework. Emerging from our research and discussion is a set of critical questions about whether or not the political moment in Canada with respect to re-invigorating recovery should be embraced, versus a rejection of the concept of recovery as too limiting in its scope and too vulnerable to professional co-optation.
\end{abstract}

"Recovery is not a concept that I really relate to because I don't think that I'm recovering from my life experiences, I'm incorporating them. I'm not surviving, I'm becoming. " - World Café Participant

Correspondence Address: Marina Morrow, Faculty of Health Sciences, Centre for the Study of Gender, Social Inequities and Mental Health, Simon Fraser University. 11514 Blusson Hall, 8888 University Ave, Burnaby, British Columbia V5A 1S6, Canada. Tel.: +(778)782-6906, Email:mmorrow@sfu.ca 
The idea of recovery originally had its roots in the psychiatric survivor movement. Yet while this movement has had empowerment and resistance to the dominance of psychiatry as its main goals, the concept of recovery has since shifted in recent years. This has led to vigorous discussion about the usefulness of the concept, and about its uses in the context of the increasing authority of biomedicalism and neoliberal policy regimes (Jacobson, Farah \& The Toronto Recovery and Cultural Diversity Community of Practice, 2010; Mental Health "Recovery" Study Working Group, 2009; Morrow, Wasik, Cohen, \& Perry, 2009; Morrow, in press; Poole, 2001; Rossiter \& Morrow, 2009). Thus, a Canadian debate is emerging that is concerned with the ways in which recovery has become distanced from its roots in psychiatric survivor activism, and its role in an evolving mental health system is being questioned (Poole, 2011).

In our view, central to any discussion about recovery must be recognition of the profound discrimination faced by people who have been psychiatrized, and the connections between recovery and the social and structural barriers that shape, facilitate or impede recovery. These social and structural aspects are articulated and enacted through a number of dimensions of power such as biomedicalism, racialization, sanism ${ }^{2}$, sexism, ageism, heterosexism, etc., calling out for an intersectional social justice analysis of recovery. That is, an analysis that foregrounds an understanding of power as it is distributed in the mental health care system, and the accompanying interlocking forms of oppression through which it operates (Burman, 2004; Burman \& Chantler, 2003; LeFrancois, 2011; Rossiter \& Morrow, 2011).

In this paper we set out the context in which experiences of mental distress occur with an emphasis on the contributions of social and structural factors and then make a case for the use of intersectionality as an analytic and methodological framework for understanding these factors. Intersectionality is a theory which can be used to examine the ways in which various social and cultural categories (such as race, sex, class, etc.) intertwine, as well as the relationships between them (Crenshaw, 1991; Knudsen, 2006). Thus, it is a powerful tool for exploring the various social, political and economic processes through which people experience oppression and privilege (Hankivsky, 2011).

We then turn to the political urgency for taking up the concept of recovery and argue for the importance of research and practice that addresses professional domination of the field, and that promotes ongoing engagement and dialogue about recovery as both a personal and social experience. To this end, we describe a unique project that sought to deepen our understanding of how recovery is being thought about and applied in the current context of mental health care in Vancouver, BC, with a specific focus on how, and whether, people are taking up and addressing dimensions of power that we see as critical to the operationalization of recovery within a social justice framework. Emerging from our research and discussion is a set of critical questions about whether or not the political moment in Canada with respect to re-invigorating recovery should be embraced, versus a rejection of 
the concept of recovery as too limiting in its scope and too vulnerable to professional co-optation. In this respect, our work highlights a number of key issues, such as: Given the dominance of the biomedical paradigm in mental health and the focus of resources on acute and psychiatric care, how can the social and structural barriers faced by psychiatrized individuals be addressed in recovery? If the participation of people with lived experience in mental health system reform, as advocates and peer support workers, is one of the cornerstones of a recovery-oriented system, what does it mean when peers face ongoing discrimination and become co-opted by professionalism? Finally, but perhaps most significantly, drawing on our research participants' call for a social justice framework in mental health, we consider the components of an intersectional social justice framework and the possibilities this has to offer vis-à-vis recovery and a transformed mental health care system.

\section{Mental Health and Social and Structural Inequities}

Experiences of mental illness and distress, regardless of their origins, take place in a social, cultural and historical context (e.g., Hacking, 2002; Porter, 2002) which includes environments of discrimination that are structured through legal, medical and psychological practices and policies. These practices and policies play out in distinct ways for different groups within society. There are now substantive bodies of literature which illustrate the ways in which the practices of psychiatry have served historically to pathologize some groups of people (e.g., women, racialized peoples, people living in poverty) over others (Baker \& Bell, 1999; Caplan, 1995; Caplan \& Cosgrove, 2004; Metzl, 2009; Van Os, Kenis, \& Rutten, 2010). This has resulted in, for example, psychiatric diagnoses being disproportionately applied to certain groups (e.g., schizophrenia to black men) and the psychiatrization of women's normal life experiences such as the post partum period and menopause (Metzl, 2009; Ussher, 1991; 2011).

Researchers have further documented the effects of social inequities such as homelessness, racism, colonialism and poverty on mental health, both with respect to exacerbating existing distress and/or creating distress (Boyer, $\mathrm{Ku}, \&$ Shakir, 1997; Kirmayer, Brass, \& Tait., 2001; Mental Health Commission of Canada, 2009). Furthering the literature on stigma against people diagnosed with "mental illness" is a burgeoning scholarship which argues that diagnoses and labels of mental illness themselves constitute a form of inequity. This is referred to as "sanism," (Birnbaum, 2010; Fabris, 2011; Ingram, 2011; Perlin, 2000) or in LeFrancois's (2011) terms, "psychiatrization," which she sees as the practice, or result, of sanism. Sanism and psychiatrization are thus used to understand the discrimination against people diagnosed with mental illness, but also go further in their aim to unsettle assumptions about rationality, normality and madness.

Finally, more medically oriented literature has made the argument that certain groups in society are more vulnerable to mental illness and are thus at 
risk of not receiving services and supports (Patterson, Somers, McKintosh, Shiell, \& Frankish, 2008; Standing Senate Committee on Social Affairs, Science and Technology, 2009). Taken together, these varied ways in which social and structural inequities in mental health operate tell us something about the ways in which power is distributed in the mental health system. Chief among these forms of power is biomedicalism and the pharmaceutical industry, which when coupled with the dramatic erosion of the social welfare system in Canada over the past 15 years, has led to a system that rations resources based on diagnosis and severity of symptoms, and responds primarily through medication and medication management over and above social supports and responses. Biomedicalism also operates discursively within society to ensure that the dominant way of understanding distress is through the lens of neurobiology, eclipsing all other possible frameworks and approaches. We counter this trend with a call towards applying the analytic lens of intersectionality to the study of and amelioration of social and structural inequities in mental health. Intersectionality involves the examination of the social, political and economic processes through which oppression and privilege are experienced by individuals (e.g., LeFrancois, 2011; Rossiter \& Morrow, 2011). As asserted by many, we see intersectionality as necessarily coupled with a social justice framework, which understands social and health inequities to be about differential access to power and resources (BurgessProctor, 2006; Collins, 1990; Hankivsky, \& Cormier, 2009).

Although in recent years mental health has gained a profile on the national policy agenda, the social and structural aspects of mental health continue to be marginalized as do the voices of people with lived experience of mental distress, especially those that challenge psychiatry. It is in this context that recovery is being re-invigorated and to which we turn to next.

\section{Recovery as Political Exigency}

Recovery as a concept and as a framework for guiding the mental health care system is currently being re-imagined and re-animated in the Canadian context. Following several decades of focus on health reform, Canada as a nation has only recently turned its attention to mental health with a focus on scrutinizing the existing mental health service system (see Kirby, 2006), the outcome of which has been the establishment of a Mental Health Commission which had a mandate to develop a national framework and strategy for mental health (Mental Health Commission of Canada, 2009). The establishment of a recovery-oriented mental health system is the cornerstone of the strategy, which was released in the Spring of 2012 (Mental Health Commission of Canada, 2012). Thus, recovery as a concept and as a guiding framework for mental health care must be addressed with some urgency as policy decision makers and mental health planners and providers are primed to integrate recovery into the mental health care system and/or re-invigorate existing recovery-oriented programming and practice. 
Recovery has been conceptualized in a myriad of ways but in general the literature and discussion of recovery falls into three camps: that which emphasizes recovery as a personal journey; that which addresses the social aspects of recovery; and that which rejects the concept of recovery outright for its contribution to both sanism and the process of psychiatrization. The first camp has dominated the conceptualizations and practice of recovery in the mental health care system and too easily plays into purely biomedical understandings of mental illness (Morrow, in press). That said there is an emergent discussion of recovery in the context of rights, anti-oppressive practice and intersectionality, all of which have social justice as their goals. On the issue of rights there is a long tradition within psychiatric survivor activism of identifying the ways in which the rights of individuals and psychiatrized people as a group have been breached (Fabris, 2011). Some of this work has been integrated into discussions about recovery (Mental Health "Recovery" Study Working Group, 2009; Repper, 2011). Repper, for example, writing in the UK context looks at recovery and social inclusion using a civil rights frame, and focuses on the idea that everyone-including those deemed "mentally ill" - have the right to determine the course of their own lives, regardless of whether or not they are regarded to possess insight (2011).

Another promising approach is that which is emerging from intersectionality frameworks and anti-oppressive practice. Although these approaches have not been widely adopted within the mental health system (Rossiter \& Morrow, 2011; van Mens-Verhulst \& Radtke, 2008) there is some evidence to suggest that they could be applied to discussions of recovery (Burman, 2004; Poole, 2011; Rossiter \& Morrow, 2011; van Mens-Verhulst \& Radtke, 2008). Anti-oppressive practice bears much in common with intersectionality approaches but it emerges from social work and is focused primarily on how to engage with social and structural inequities in practice from the perspective of providers (Poole, 2011); it may or may not operationalize an intersectional framework. Like intersectionality, however, anti-oppressive practice recognizes the role of structural and systemic barriers in differentially shaping the lives of groups and individuals. Missing, however, from both frameworks is an explicit recognition of sanism as a form of oppression, that is, the valuing of rational thinking and socially acceptable forms of behavior, and the subsequent ostracization and/or punishment of people who do not or cannot conform. Some argue that sanism must be seen as a form of oppression akin to that of sexism or racism (Ingram, 2011; LeFrancois, 2011), however, more analytic work is required to think through the varied ways in which sanism systematically oppresses people.

In the Canadian context, with some exceptions, (Jacobson, Farah, \& The Toronto Recovery and Cultural Diversity Community of Practice, 2010; Mental Health "Recovery" Study Working Group, 2009) the research literature has not substantively investigated what people with lived experience of psychiatrization have to say about recovery and its meaning vis-à-vis their lives and connections to the mental health care system. Further, 
recovery as a paradigm for mental health care system transformation has only recently begun to be discussed and empirically investigated (O'Hagan, 2004; Morrow, Pederson, Smith, Josewski, Jamer, \& Battersby, 2010; Myers, 2010; Piat \& Subetti, 2009) and questions remain as to whether recovery is the best conceptual framework for system change. The research we present next constitutes an attempt to begin to redress these gaps and further the dialogue on recovery as it is occurring in contemporary mental health practice.

\section{The Research}

Our project began with the formation of a research team that included people who identified as having had lived experience of mental distress and use of the mental health care system; health policy decision makers in our local health authority; service providers; and academics, all of whom had an interest in exploring social inequities in mental health recovery (Morrow, Jamer, \& Weisser, 2010). The collaborative research team was intentionally sought to help build connections between people differently positioned in relation to the mental health care system, with the idea that this would generate beneficial discussions as well as useable research outcomes. The purpose of the study was to explore conceptualizations of recovery in the mental health field that addressed social and structural inequities in mental health, with a particular interest in foregrounding people's lived experiences of mental health issues and system use. The study involved a scoping review of the literature (Arksey \& O'Malley, 2005), a World Café (Brown \& Isaacs, 2005), and creative knowledge exchange.

Scoping review methodology, "aim (s) to map rapidly the key concepts underpinning a research area and the main sources and types of evidence available, and can be undertaken as stand-alone projects in their own right, especially where an area is complex or has not been reviewed comprehensively before" (Mays, Roberts, \& Popay, 2001, p. 194). A scoping review of mental health and other relevant literatures was conducted in order to identify current definitions, models, and conceptualizations of recovery and to explore recovery in its intersections with social inequities (Weisser, Morrow, \& Jamer, 2011).

World Café methodology involves concurrent round table discussions which are focused around a set of questions of relevance to the issue being explored. Brown \& Isaacs (2005) refer to this approach as "conversations that matter," in part because it allows for multi-layered discussions which build upon one another, and fosters the expression of multiple perspectives and kinds of knowledge (in this case personal, professional, academic).

Our World Café was structured around four discussion questions, each relating to different aspects of the team's interest in mental health and social inequities (Morrow, Jamer, \& Weisser, 2010). The questions evolved out of dialogue among the research team members, over a number of meetings and included: 1) What are some of the social and structural barriers that impact 
people's mental health recovery?; 2) What are the strengths and weaknesses of current mental health recovery models with respect to addressing social and structural inequities?; 3) What would components of a recovery model look like that integrated social and structural inequities and how would we get there?; 4) How can people's experiences with mental health issues inform the development and practice of recovery? What would it take to support and implement this model?

The World Café participants included twenty four mental health and social service front line workers; mental health and addictions managers; policy makers; people with lived experience of mental distress; family members; and community leaders engaged in mental health work. Although many participants wore several hats (such as service user and service provider, family member and policy maker, etc.), about half of the participants identified as having lived experience, and about half were service providers, managers, or policy actors in the mental health field. Throughout the World Café participants moved from table to table, not as a group but in different formations each time, to ensure that everyone attending would come into contact with each other at some stage of the process. Each discussion table had a facilitator and a note taker who recorded and tracked the emerging conversations. The notes from the World Café were organized thematically and then analyzed in order to begin to identify some of the key components of a mental health care system that would be informed by multiple perspectives and would be responsive to social and structural inequities.

The last stage of the project involved creative knowledge exchange through the development of a series of four skits illustrating each of the four topics mentioned above. The skits were linked with some analysis, quotes from the World Café, and a discussion of the findings for presentations to relevant audiences (Morrow, Jamer, Weisser, Willow, \& Omura, 2011a; Morrow, Jamer, Weisser, Willow, \& Omura, 2011b; Morrow, Jamer, Weisser, Willow, Omura, \& Ingram, 2011).

The four skits were respectively titled, "Revolving Door," "The Multiple Meanings of Recovery," "Round Peg, Square Hole" and "Medical Model." Each skit highlighted an aspect of the current mental health care system in $\mathrm{BC}$ to which practitioners and service users could relate. For example, the "Revolving Door" skit shows how the system is currently crisis oriented and the ways in which services (both psychiatric and social) are tied to psychiatric diagnosis and level of severity, which acts as a disincentive towards recovery for some service users for fear that getting better will mean losing housing, disability benefits or other valuable supports. "Round Peg, Square Hole" illustrates the ways in which the mental health care system is largely unable to deal with the complexities of people's lives. People often have to seek services and supports from a variety of places rather than being able to have a range of needs met within all services. In what follows, we detail both the findings from our scoping review and from the World Café noting those of relevance to our contention that an intersectional social justice framework is needed for mental health system transformation. 


\section{The Findings}

Recovery in the literature: The scoping review

In order to surface literature that might tell us something about the intersections between social and structural inequities in their relation to recovery, a scoping review of the literature was conducted. The literature reviewed was from 1980 onward and was from Canada, the United States, the United Kingdom, Australia, or New Zealand. Using purposive sampling and a search of social science databases, both peer-reviewed and grey literature (e.g., non-published reports and project descriptions) was reviewed in order to identify current models and frameworks for mental health recovery. Attention was paid to whether, and to what degree, existing literature addressed social and structural inequities in mental health and the degree to which the development, application and evaluation of recovery models are professionally versus experientially driven (Weisser, Morrow, \& Jamer 2011).

Emerging from the literature review were several broad themes, including: the concept of recovery as potentially oppressive or harmful; the usefulness (or lack thereof) of the term recovery; the concept of second class citizenship for people diagnosed with mental illness; recovery as a cornerstone of mental health system transformation; and the need for a framework that makes use of the social determinants of health and/or the social model of disability. Finally, there is an emerging conversation about social justice in mental health.

There are two different schools of thought in the current literature about why the concept of recovery may be harmful. The first is the idea that recovery offers a false sense of hope, that it implies a return to the pre-illness self, and is therefore misleading (Whitwell, 1999). Peyser (2001) argues that recovery is not possible for those who are extremely mentally ill, and that concepts such as empowerment cannot help those who are completely held hostage by their "illnesses." According to Peyser, people who "lack insight" are simply unable to effectively use the tenets of recovery (2001). In contrast to this viewpoint are those who accuse recovery of being either a passing trend or a cash grab - a way to download responsibility from the state to the service user (Davidson, O'Connell, Tondora, Styron, \& Kangas, 2006; Dickerson, 2006; Ridgway, 2001). That is to say, the imperative to recover is viewed simply as an extension of the neo-liberal agenda (Morrow, in press) and a call for citizen productivity (Myers, 2010).

This literature, from two different philosophical positions, raises questions about the usefulness of the concept of recovery-some authors, for example, suggest that recovery cannot be realized in the context of discrimination (Myers, 2010), while others feel that the concept is inadequate in the context of the medical realities of mental illness (Peyser, 2001; Whitwell, 1999). Myers (2010) also discusses the concept of the mental health service user as "second class citizen," one to whom much-needed cultural capital is denied (p.300). He speaks specifically of those who are disenfranchised socially and economically to the point where the general tenets of recovery are no 
longer possible or available to them (Myers, 2010). Myers' work is consistent with the many personal narratives of psychiatrized people who document the ways in which they have been oppressed and marginalized by the system (Blackbridge, 1997; Blackbridge \& Gilhooly, 1985; Capponi, 1992, 1997, 2003; Fabris, 2011; Shimrat, 1997).

Literature on mental health system transformation focuses not just on individuals but on the ways in which the system as a whole can become recovery-oriented (Adams, Daniels, \& Compagni, 2009; Friedli, 2009; Mental Health Commission of Canada, 2009; O'Hagan, 2004; Piat \& Subetti, 2009). Although this process has not yet been fully documented or empirically studied in most regions, there are some promising signs for its success. For example, New Zealand as a nation has perhaps gone the furthest in terms of moving away from individual conceptualizations of recovery towards one that views cultural (re)integration as recovery, and has applied this concept directly to system change (O'Hagan, 2004). So, for example, with the support of its Mental Health Commission, New Zealand has had success in educating its mental health workers about culturally diverse perspectives on mental health, including recovery.

In the literature, social inequities were rarely mentioned, and where mentioned race, ethnicity, immigration, and culture were privileged above other types of inequities such as disability, age or sexual orientation. Additionally, when issues such as racism were mentioned, it was almost always in the context of the individual's struggle with racism; that is to say, racism as a structural and systemic problem was rarely discussed. Even literature that did focus on structural barriers, culturally appropriate services, or an overhaul of the mental health system (Jacobson, Farah, \& The Toronto Recovery and Cultural Diversity Community of Practice, 2010; O'Hagan, 2004), did not go so far as to address the overlapping and intersectional nature of oppression in mental health or the complex relationship between social inequities and recovery.

For example, some excellent Canadian work has been done which focuses in on the cultural relevance of the concept of recovery. Jacobson, et al., (2010) identify a "Culturally-Responsive Model of Recovery" (p.19) which places the individual in context (family, community, geography, culture, oppression/ privilege, social determinants of health, history, etc.) but their analysis discusses each form of oppression separately rather than as interlocking. Almost all of the literature tends to address one aspect of social inequity at a time in its relationship to recovery. So for example, O'Brien and Fullagar (2008), address gender; Myers (2010), addresses class; Lapsley, Nikora, and Black (2002), Jones, Hardiman, and Carpenter (2007), Ida (2007), and Armour, Bradshaw, and Roseborough (2009) all address culture; and Daley (2010), addresses sexual orientation. Thus, the recovery literature mimics what is found in the mental health literature more generally, that is to say that the literature tends to treat social processes as variables that can be used as discrete categories of analysis. The literature also tends to privilege certain categories over others (e.g., gender and culture are privileged over sexual 
orientation) (Rossiter \& Morrow, 2011). Despite this there is some evidence, as discussed earlier, of an emergent literature which addresses rights and social justice as components of recovery (Mental Health "Recovery" Study Working Group, 2009; Repper, 2011).

\title{
The World Café
}

In this section we discuss the themes emerging from the World Café that focused on intersectional issues such as: power and control in mental health; social and structural inequities; the co-optation of peer workers in mental health; and social justice.

Social and structural inequities: Addressing power and control.

\begin{abstract}
I don't see the connection between recovery and the mandates of mental health teams. It's kind of like a self-fulfilling prophecy, because if the mental illness must be "serious and persistent" in order to get funding, how can a person "recover" from it? (World Café participant)
\end{abstract}

The system has been very limited in the way it understands mental health. Recovery goes beyond the medical model, in that it includes the medical model but it can include much more. It [recovery] is more holistic and goes away from the reductionist understanding of mental health. (World Café participant)

The quotes above reflect a common theme emerging from the World Café, that is, that people who are entrenched in the mental health system often have very little control over their own lives, which necessarily makes recovering difficult or impossible. Participants noted often that the mental health care system and associated support systems are designed to perpetuate themselves, by upholding people's dependence upon them. Many participants, for example, spoke about the ways in which the mental health system rewards pathology, and how it is oriented towards crisis rather than prevention, leaving those to fall through the cracks who are more stable but still need help. Participants felt that if there were adequate treatment resources available to everyone who needed them, people might feel freer to get better, as they would not be worried about the possibility of losing their support systems and access to treatment. In this vein, numerous examples of the ways in which policies and bureaucratic practices work against recovery were discussed, with a focus on poverty, disability benefits, service mandates, and resource constraints.

Thus, by using local examples, participants illustrated the ways in which social assistance and disability benefits are set up so as to trap people in cycles of dependence and poverty, rather than assisting them to move forward with their lives when they are ready and able to do so. For example, the prohibition against accumulation of assets when on disability benefits or income assistance, and restrictions related to the amount one can earn 
in paid work while on benefits, were cited as formidable barriers in terms of people being able to improve their financial situations and also as a discouragement from seeking employment. This combined with substantial barriers to employment (lack of workplace accommodations for mental health disabilities, discrimination, etc.,) form insurmountable obstacles.

Several issues related to service mandates and resource constraints were raised during the World Café. It was noted that some people are excluded from receiving much-needed treatment because they do not fall into the increasingly crisis-oriented mandates that organizations must follow. Similarly, World Café participants noted that the disconnect between different services/sectors can negatively impact recovery; for example, a 30 day in-patient hospital stay can result in a loss of housing, which can impact a person's ability to get well.

\section{The corporatization of peers}

At the World Café we found that participants were extremely interested in discussing how people with lived experience might play more meaningful roles in policy development and peer support positions. The World Café participants focused their discussion on those who work in mental health, such as peer support workers and facilitators, peer specialists, peer researchers, advocates, consultants, and board members. The discussion centred around ways in which these roles could move beyond tokenism and instead involve the full integration of peers into the mental health system, in a way that is meaningful both to them and to the people they are supporting.

Historically, peer support workers within mental health have been poorly compensated and typically hired on short term contracts ${ }^{3}$. Many peers are being integrated into pre-existing bureaucratic structures; however, problems exist in terms of genuine integration. Many of these problems have to do with the system itself, and the ways in which these structures and their practices may not always be comfortable for all peers.

In relation to this, one participant in the World Café coined the phrase "the corporatization of peers," and another the "loss of peerness," to describe how the peer role has been co-opted by professionalism over time, descriptions which were eagerly taken up by many others at the table. These terms were used to describe both the subtle, and not so subtle, ways in which peer support workers who are part of mental health teams or who work in hospitals are encouraged to act "normal," professional, or well socialized - thus losing the very essence of what makes them peers in the first place. Many participants argued that this need to appear professional creates distance between the peer support worker and the person they are supporting. Additionally, many peer support workers are expected to uphold the values of the medical model, regardless of whether they or the person they are supporting agree with this model. 
A social justice approach to mental health

In the World Café discussions, participants raised the idea of a social justice approach to mental health. Specifically, this conversation was tied to discussions of the ways in which psychiatrized people were viewed by society and discriminated against. So, for example, participants wanted society to become more accepting of the full expression of the human condition and experience, rather than viewing people solely through a biomedical lens which some felt unduly pathologized people. Further, some participants pointed out the fact that social expectations of mental health get taken up in conceptualizations of recovery, creating standards about what a recovering person should look and act like. Among other comments were discussions about the role of the pharmaceutical industry in propping up narrow biomedical definitions of mental health, and a call for more expansive ideas about what constitutes knowledge about mental health, with a focus on the role of lived experience.

Despite widespread enthusiasm at the World Café for frameworks of recovery that were rooted in notions of social justice, it was our observation that participants often struggled with framing recovery in terms of social and structural inequities. Although social justice was named, there was a tendency for people to revert back to more individualistic framings of recovery, or to discussions about specific barriers in the system, rather than to understand these barriers as structurally produced and differentially experienced. The one exception was that participants were able to articulate how individual notions of recovery may not resonate with people from non-dominant, ethno-racial groups. For example, some cultures value collectivity over individuality, or inter-dependence over independence, as well as hold a place for unique behaviors in a way that contemporary Western society does not. In these instances culture was still viewed more as an individual attribute and discussions of how systemic racism and/or ethnocentrism might be at play in the mental health system were not raised (Morrow, Jamer, \& Weisser, 2011). Thus, participants themselves were so embedded in the current mental health system and its discursive practices that they appeared to struggle to move away from individualistic framings, even as they began to name forms of discrimination such as racism, sexism and - importantly—sanism, in relation to recovery. This is not surprising given how few spaces currently exist to discuss the social and structural aspects of mental health. Further, we observed that even in the context of our World Café the discussion became polarized between the role of the biomedical and the role of the social in mental health, and around the needs and concerns of different "stakeholders." The latter was most obvious with respect to debate about the role of families, especially with respect to making treatment choices on behalf of their family members. As a way of untangling these tensions and challenges we suggest that one method of framing recovery is to attend to social and structural inequities through the lens of intersectionality (Morrow, Jamer, \& Weisser, 2011). Intersectionality, for example, would allow the power dynamics in families 
to be explored, and would surface the ways in which loss of rights for people diagnosed with mental illness are a part of those dynamics that cannot be ignored or eclipsed by discourses of care and treatment. In general, using the framework of intersectionality allows a way out of polarizing the biomedical and the social, by revealing that people's "mental illness symptoms" might be linked or produced in simultaneous ways with conditions of poverty, homelessness, and other forms of inequity (Weber, 2006). Intersectionality also allows for an analysis of the ways in which power is at play in the mental health care system. The driving force behind this viewpoint is "the pursuit of social justice" (Weber, 2006). In summary, our findings suggest that service providers and users in the mental health care system struggle to resist the idea of recovery as a personal journey, but are often thwarted in these attempts due to the systematization of biomedicalism. The authority of biomedicalism works to undermine broader social and structural understandings of recovery. It also reflects a sanist ethic in its treatment of people in the mental health system and of the peers who are trying to support them.

\section{An Intersectional Social Justice Framework for Mental Health Recovery}

In our view to answer the World Café question, "what would it take to transform the mental health care system and does recovery have a role in this transformation?', a reformulation of recovery as embedded in particular social and structural contexts which foreground an intersectional social justice approach is required. The components of such an approach are as follows:

- Recognize and address the ways in which active discrimination against people diagnosed with mental illness is systemic, and the ways in which this is compounded by other experiences of oppression (e.g., sexism, racism, ethnocentrism, ageism, classism, heterosexism). Thus, sanism must be understood as a key critical analytic lens for understanding mental distress.

- Psychiatric survivors must be at the forefront in leading the re-invigoration of recovery as it was originally characterized by the psychiatric survivor movement, and in so doing, develop new structures and ways of organizing the mental health care system so that "peerness" can be retained.

- Rebalance the mental health care system to address both the biomedical and social needs of people. Specifically, we need to:

1. Enact changes to the social welfare system that would allow for people to break the cycles of poverty and dependence. This would include: i) raising the rates for social assistance and disability benefits to bring them in line with the cost of living; ii) allowing people on disability benefits to hold assets and have asset accumulation; iii) allowing people more flexibility to go on and off disability benefits. 
2. Challenge biomedicalism and open up a discursive space for discussion about mental illness that does not preclude the biomedical, but that allows for a wider range of understandings and approaches.

As Canada moves towards adopting a recovery framework for mental health it will be critically important for regions and communities to actively engage in "recovery dialogues" and to support the active leadership of psychiatric survivors in this process. In this way we can begin to re-capture the roots of recovery in claiming the humanity of psychiatrized people, expose the abuses of psychiatry, and prove that people whose lives are marked by distress can continue to live full and meaningful lives with dignity. It is clear from our work that active discrimination against people with mental illness is systemic, that sanism continues without being questioned and that much work needs to be done in order to incorporate a social and structural analysis of mental health and recovery into the current mental health care system.

Further, recovery without a full recognition of the current social and political context which has eroded social welfare supports will be impotent to foster real systemic change. Thus, mental health providers, advocates and decision makers must strongly resist further trends toward biomedicalism. This does not mean a rejection of the role of biology in mental health, but rather, recognition that the biological occurs within a social context that involves interlocking forms of oppression which impact mental health and erode social justice. Only if these conditions are met can we truly seize the political moment for re-invigorating, and we would argue, re-inventing recovery.

\section{Notes}

1 The authors would like to thank the Centre for the Study of Gender, Social Inequities and Mental Health, writing group and particularly, Brenda Jamer and our two anonymous reviewers, for their thoughtful comments and editorial assistance on earlier drafts of this paper.

2 Sanism refers to the irrational societal prejudice against persons who are labelled as "mentally disabled" (Perlin, 2003).

3 This has begun to shift in some jurisdictions where new jobs are being advertised that are unionized and include full benefits.

\section{References}

Adams, N., Daniels, A., Compagni, A. (2009). International pathways to mental health transformation. International Journal of Mental Health, 28(1), 30-45.

Armour, M. P., Bradshaw, W., \& Roseborough, D. (2009). African Americans and recovery from severe mental illness. Social Work in Mental Health, 7(6), 602-622.

Arksey, H., and O'Malley, L. (2005). Scoping studies: Towards a methodological framework. International Journal of Social Research Methodology, 8(1), 19-32.

Blackbridge, P. (1997). Prozac highway. Vancouver: Press Gang.

Baker, F.M., Bell, C.C. (1999) Issues in the psychiatric treatment of African Americans. Psychiatric Services, 50(3), 362-68. 
Birnbaum, R. (2010) My father's advocacy for a right to treatment. Journal of the American Academy of Psychiatry Law, 38(1), 115-123.

Blackbridge, P., Gilhooly, S. (1985). Still sane. Vancouver: Press Gang.

Boyer, M., Ku, J., \& Shakir, U. (1997). The healing journey: Phase II report-women and mental health: Documenting the voices of ethnoracial women within an anti-racist framework. Toronto: Across Boundaries Mental Health Centre.

Brown, J., \& Isaacs, D. (2005). The World Café : Shaping our futures through conversations that matter. San Francisco, CA: Berrett-Koehler Publishers.

Burgess-Proctor, A. (2006). Intersections of race, class, gender, and crime: Future directions for feminist criminology. Feminist Criminology, 1(1), 27-47.

Burman, E., Chantler, K. (2003). Across and between: Reflections on researching "race" gender and mental health. Feminism and Psychology, 13(3), 302-309.

Burman, E. (2004). From difference to intersectionality: Challenges and resources. European Journal of Psychotherapy, Counselling and Health, 6(4), 293-308.

Capponi, P. (1992). Upstairs in the crazy house. Toronto: Viking.

Capponi, P. (1997). Dispatches from the poverty line. Toronto: Penguin.

Capponi, P. (2003). Beyond the crazy house: Changing the future of madness. Toronto: Penguin.

Caplan, P (1995) They Say You're Crazy: How the World's Most Powerful Psychiatrists Decide Who's Normal New York: Perseus Books.

Caplan, P., \& Cosgrove, L. (Eds). (2004) Bias in psychiatric diagnosis. Maryland: Roman \& Littlefield Publishing.

Collins, P. H. (1990). Black feminist thought: Knowledge, consciousness, and the politics of empowerment. Boston: Unwin Hyman.

Crenshaw, K. (1991). Mapping the margins: Intersectionality, identity politics, and violence against women of colour. Standford Law Review, 43(6), 1241-1299.

Davidson, L., O’Connell, M., Tondora, J., Styron, T., \& Kangas, K. (2006). The top ten concerns about recovery encountered in mental health system transformation. Psychiatric Services, 57(May), 640-645.

Dickerson, F. B. (2006). Disquieting aspects of the recovery paradigm. Psychiatric Services, 57(May), 647-647.

Fabris, E. (2011). Tranquil prisons: Chemical incarceration under Community Treatment Orders. Toronto: University of Toronto Press.

Friedli, L (2009). Mental health, resilience and inequalities. Geneva: WHO. http://www. mentalhealthpromotion.net/resources/mental-health-resilience-and-inequalities.pdf

Hacking, I. (2002) Mad travelers: Reflections on the reality of transient mental illnesses. Boston: Harvard University Press.

Hankivsky, O. (Ed.). (2011). Health inequities in Canada: Intersectional frameworks and practices. Vancouver: UBC Press.

Hankivsky, O., \& Cormier, R. (2009). Intersectionality: Moving women's health research and policy forward. Vancouver, BC: Women's Health Research Network.

Ida, D. J. (2007). Cultural competency and recovery within diverse populations. Psychiatric Rehabilitation Journal, 31(1), 49-53.

Ingram, R. (2011). Recovery from compulsory sanity. Presentation for Society for Disabilities Studies Conference, San Jose, California.

Jacobson, N., Farah, D., \& The Toronto Recovery and Cultural Diversity Community of Practice. (2010). Recovery through the lens of cultural diversity. Toronto, Ontario, Canada: Community Resource Connections of Toronto; Centre for Addiction and Mental Health; Wellesley Institute.

Jones, L. V., Hardiman, E. R., \& Carpenter, J. (2007). Mental health recovery: A strengthsbased approach to culturally relevant services for African Americans. Journal of Human Behavior in the Social Environment, 15(2-3), 251-269.

Kirby, M. (2006). Out of the shadows at last: Transforming mental health, mental illness and addiction services in Canada, Final Report of the Standing Senate Committee on Social Affairs, Science and Technology.

Kirmayer L., Brass G.M., Tait C.L. (2001). The mental health of Aboriginal peoples: Transformations of identity and culture. Canadian Journal of Psychiatry, 45(7), 607-617.

Knudsen, S. (2006). Intersectionality - A theoretical inspiration in the analysis of minority cultures and identities in textbooks. In E. Bruillard, B. Aamotsbakken, S. Knudsen, \& M. 
Horsley (Eds.), Caught in the Web or Lost in the Textbook? Eight International Conference on Learning and Educational Media (pp. 61-76). The International Association for Research on Textbooks and Educational Media. Retrieved from http://www.iartem.no/documents/ caught_in_the_web.pdf

Lapsley, H., Waimarie Nikora, L., \& Black, R. (2002). “Kia mauri tau!' Narratives of recovery from disabling mental health problems. Wellington, New Zealand: University of Waikato Mental Health Narratives Project/Mental Health Commission of New Zealand.

LeFrancois, B.A. (2011). Queering child and adolescent mental health services: The subversion of heteronormativity in practice. Children and Society. Published online on 26 APR 2011 at doi: 10.1111/j.1099-0860.2011.00371.x

Mays, N., Roberts E., \& Popay, J. (2001). Synthesizing research evidence. In N. Fulup, P. Allend, A. Clarke, \& N. Black (Eds.), Studying the organisation and delivery of health Services: Research methods (pp. 188-220). London: Routledge.

Mental Health "Recovery" Study Working Group. (2009). Mental health 'recovery': Users and refusers. Toronto: Wellesley Institute.

Mental Health Commission of Canada (2012) Changing Directions, Changing Lives: The Mental Health Strategy for Canada. Ottawa: Mental Health Commission of Canada.

Mental Health Commission of Canada. (2009). Toward recovery \& well-being: A framework for a mental health strategy for Canada. Calgary, AB: Mental Health Commission of Canada.

Metzl, J. (2009) The Protest Psychosis: How Schizophrenia became a Black Disease. Boston: Beacon Press.

Morrow, M., Frischmuth, S., \& Johnson, A. (2006). Community based mental health services in $B C$ : Changes to income, employment and housing security. Vancouver, Canadian Centre for Policy Alternatives.

Morrow, M., Pederson, A., Smith, J., Josewski, V., Jamer, B., \& Battersby, L. (2010). Relocating mental health care in British Columbia: Riverview Hospital redevelopment, regionalization and gender in psychiatric and social care. Vancouver: Centre for the Study of Gender, Social Inequities and Mental Health.

Morrow, M., Wasik, A., Cohen, M., \& Perry, K. (2009). Removing barriers to work: Building economic security for people with mental illness. Critical Social Policy, 29(4), 655-676.

Morrow, M. (in press). Recovery: Progressive paradigm or neoliberal smokescreen? R. Menzies, G. Reaume, \& B. Lefrancois, (Eds.), Mad Matters: A Critical Reader in Canadian Mad Studies. Toronto: Canadian Scholar's Press.

Morrow, M., Jamer, B., \& Weisser, J. (2010). The recovery dialogues: A critical exploration of social inequities in mental health recovery. Vancouver: Centre for the Study of Gender, Social Inequities and Mental Health. http://www.socialinequities.ca/wordpress/wp-content/ uploads/2011/02/The-Recovery-Dialogues-Team-Report.Final_.style_.pdf

Morrow, M., Jamer, B. \& Weisser, J. Willow, b., \& Omura, C. (2011a) The recovery dialogues: A critical exploration of social inequities in mental health recovery. Presented to the Vancouver Coastal Health Authority March 2 \& January 27, 2011 Vancouver, BC.

Morrow, M., Jamer, B. \& Weisser, J. Willow, b., \& Omura, C. (2011b) Creative Knowledge Exchange: A Critical Exploration of Social Inequities in Mental Health. Presented at Fifth JEMH Conference on Ethics in Mental Health, Ethical Challenges in Service Delivery and Design May 12-14, 2011 in Kelowna, British Columbia, Canada.

Morrow, M., Jamer, B., Weisser, J. Willow, b., Omura, C., \& Ingram R. (2011) Recovering "recovery" in mental health: A critical feminist intersectional approach. Presentation for beyond access: From disability rights to disability justice. Society for Disability Studies June 15-18, 2011 in San Jose, California.

Myers, N. L. (2010). Culture of recovery? Schizophrenia, the United States' mental health system, and the American ethos of the self-made man. ProQuest Information \& Learning. Dissertation Abstracts International Section A: Humanities and Social Sciences, 70(8).

O'Hagan, M. (2004). Guest Editorial: Recovery in New Zealand: Lessons for Australia. Australian e-Journal for the Advancement of Mental Health, 3(1), 1-3.

O'Brien, W., \& Fullagar, S. (2008). Rethinking the relapse cycle of depression and recovery: A qualitative investigation of women's experiences. Social Alternatives, 27(4), 6-13.

Patterson, M., Somers, J., McKintosh, K., Shiell, A., \& Frankish, J. (2008) Housing and support for adults with severe addictions and/or mental illness in British Columbia. Vancouver: Centre for Applied Research in Mental Health and Addiction. 
Perlin, M, (2000) The hidden prejudice: Mental disability on trial. Washington, DC, US: American Psychological Association.

Perlin, M. (2003). "You have discussed lepers and crooks": Sanism in clinical teaching. Clinical Law Review, 9, 683-729.

Peyser, H. (2001). Commentary: What is recovery? Psychiatric Services, 52(4), 486-487.

Piat, M., Subetti, J. (2009) The development of a recovery-oriented mental health system in Canada: What the experience of Commonwealth countries tells us. Canadian Journal of Community Mental Health, 28(2), 17-33.

Picard, A. (2011, Aug. 31). Mental health strategy draft doesn't go far enough. The Globe and Mail. Retrieved from: http://www.theglobeandmail.com/life/health/new-health/andre-picard/ mental- health-strategy-draft-doesnt-go-far-enough/article2149012/

Poole, J. (2011). Behind the Rhetoric: Mental Health Recovery in Ontario. Halifax: Fernwood.

Porter, R. (2002). Madness: A brief History Oxford: Oxford University Press.

Repper, J. (2011) A Rights Based Approach to Recovery and Social Inclusion. Presentation for INTAR Conference, Challenging Our Understanding of Psychosis and Exploring Alternatives for Recovery Toronto, Nov. 3-4 2011.

Ridgway, P. (2001). Re-Storying psychiatric disability: Learning from first person recovery narratives. Psychiatric Rehabilitation Journal, 24(4), 335-343.

Rossiter, K., \& Morrow, M. (2011). Intersectional frameworks in mental health: Moving from theory to practice. In O.Hankivsky (Ed.) Health inequities in Canada: Intersectional frameworks and practices (pp. 312-330). Vancouver: UBC Press.

Shimrat, I. (1997). Call me crazy: Stories from the mad movement. Vancouver: Press Gang.

Teghtsoonian, K. (2008). Managing Workplace Depression: Contesting the Contours of Emerging Policy in the Workplace. In K. Teghtsoonian and P. Moss (Eds.), Contesting illnesses: Processes and practices (pp. 69-89). Toronto: University of Toronto Press.

Ussher, J. (1991). Women's madness: Misogyny or mental illness? Amherst: The University of Massachusetts Press.

Ussher, J.M. (2011). The madness of women: Myth and experience. London; Routledge.

van Mens-Verhulst, J., \& Radtke, L. (2008). Intersectionality and mental health: A case study IST-travelling. Retrieved from: http://www.vanmens.info/verhulst/en/wp-content/ INTERSECTIONALITY\%20AND\%20MENTAL\%20HEALTH2.pdf

Van Os, J., Kenis, G., Rutten, BP. (2010) The environment and schizophrenia. Nature 468(7321), 203-212.

Weber, L. (2006). Reconstructing the landscape of health disparities research: Promoting dialogue and collaboration between feminist intersectional and biomedical paradigms. In A. J. Schultz, \& L. Mullings (Eds.), Gender, race, class and health: Intersectional approaches (pp. 21-59). Indianapolis: Wiley.

Weisser, J., Morrow, M., \& Jamer, B. (2010). A critical exploration of social inequities in themental health literature. Vancouver: Centre for the Study of Gender Social Inequities http://www.socialinequities.ca/wordpress/wp-content/uploads/2011/02/Recovery-ScopingReview.Final_.STYLE_pdf

Whitwell, D. (1999). The myth of recovery from mental illness. Psychiatric Bulletin, 23(10), 621-622.

World Health Organization. (2008). Closing the gap in a generation: Health equity through action on the social determinants of health. Geneva: World Health Organization http://whqlibdoc.who.int/hq/2008/WHO_IER_CSDH_08.1_eng.pdf 